\title{
FUSION OF RADIAL RIBS IN CARDIIDAE (BIVALVIA: VENEROIDA): IMPLICATIONS FOR PHYLOGENETIC RECONSTRUCTION AND THE STUDY OF ITERATIVE HOMOLOGY
}

SCHNEIDER, Jay A., Dept. of Geophysical Sciences, University of Chicago, Chicago,
IL 60637 U.S.A.

To properly reconstruct the phylogeny of a taxon, it is necessary to determine homologies among taxa. The assumption that repeated structures are iterative homologues has resulted in phylogenetic confusion. Radial ribs have long been used as important taxonomic characters in bivalves, but a cladistic analysis of the Cardiidae has shown that radial ribs are not homologous across genera.

Pre-Aptian cardiids have either simple, unornamented ribs, or have lost the ribs entirely. The genus Granocardium originates in the Aptian and has large spines on every third or fourth rib interspace. This row of spines is connected by a raised striation. The interspace bearing these spines is raised relative to other interspaces. Criocardium, a subgenus of Granocardium, originates in the Upper Cretaceous and has rows of small spines, also connected by a raised striation, on the interspaces not occupied by large spines; these interspaces are not concomitantly raised.

When the character states of the large and small spines, their respective striations, ribs and interspaces are analyzed throughout the Cardiidae, it becomes apparent that adjacent pairs of ribs have fused independently on three occasions. In Cenozoic cardiids, the large spines and their striations are not between ribs but on top of them. On many taxa, the top of the rib is concave, reflecting the rib top's homology with the rib interspace of Granocardium. Furthermore, some taxa bear the remnants of Criocardium's row of small spines connected by a striation in the rib interspaces. The Maastrichtian Perucardia has an intermediate morphology, with the anterior and posterior slopes as in the Cretaceous Criocardium while the central slope has the ribbing pattern of Cenozoic cardiids.

Cladistic analysis demonstrates that Cretaceous cardiids once put into the Neogene genera Fragum and Bucardium are allied with Criocardium. Species placed in Fragum resemble that genus only in its trigonal shell shape; they share the interspace spines and several other characters witth Criocardium and belong in Profragum Badve 1977. Species placed in Bucardium on the basis of round shell shape and reduction in rib number are properly placed in Austrocardium Freneix and Grant-Mackie 1978, and share the interspace striation and other characters with Criocardium. Austrocardium is another example of rib fusion: a groove, homologous to a rib interspace, runs down the top of each rib; this accounts for the reduction in rib number.

A third example of rib fusion occurs in some species of the Cenozoic genus Hedecardium. Two ribs (each one homologous to a pair of ribs in Granocardium) undergo an additional episode of fusion, forming a single rib with two parallel rows of spines and striations on top of the rib; a single one of these ribs would therefore be homologous to four ribs on Granocardium.

Fusion of iterative homologues has been discerned in many arthropods. Pairs of metameric segments have fused, leaving individual segments with twice the number of anatomical structures usually associated with a single segment. Radial ribs are not metameric (but are iterative) since they radiate from a single point as opposed to being repeated in a linear sequence. Rib fusion operating alone would imply a fifty per cent reduction in rib number, and in Austrocardium the rib number is halved. Cenozoic cardiids have fewer ribs than their Cretaceous ancestors, but only by about twenty per cent. Fusion is only one of several processes that regulate rib number on cardiids. 